unterschiedlich aktiv sind" (Gabriele Abels, S. 94) sowie um geringes Engagement der Abgeordneten in der Europapolitik, da sie den Wählern oft unbedeutend erscheint (Gabriele Abels, S. 86; Sabine Kropp, S. 187; Werner Reutter, S. 277; Birgit Eberbach-Born, S. 312). Wichtig ist Birgit Eberbach-Borns Forderung, die Regierungen in Bund und Ländern durch die Parlamente zu mandatieren (S. 289 ff.) gemäß der Anregung des ehemaligen Bundesverfassungsgerichtspräsidenten Hans-Jürgen Papier - gegen die Rechtsprechung seines Gerichts (BVerfGE 8, S. 105 ff., S. 121).

Ein differenziertes Bild vermittelt dieses Buch mit vielen Informationen. Es gilt vor allem, doch nicht nur für die Berichte aus der Praxis. In Sachen Europa leiden die Darstellungen unter der Dynamik der Entwicklung. Da ist oft wichtiger, ob EU-Repräsentanten nicht mehr von Angela Merkels Gnade abhängen, damit der Parlamentarisierungsprozess weiter voranschreitet. Trotz dieser und anderer Anmerkungen: Es ist ein empfehlenswerter Sammelband.

Erich Röper

\title{
Der Zeitpunkt der Wahlentscheidung: neue Methoden der Messung für Wissenschaftler und Wahlkämpfer
}

Plischke, Thomas: Wann Wähler entscheiden. Abläufe von Entscheidungsprozessen und der Zeitpunkt der Wahlentscheidung (Studien zur Wahl- und Einstellungsforschung, Bd. 26), Nomos Verlagsgesellschaft, Baden-Baden 2014, 334 Seiten, € 59,-.

Thomas Plischke stellt in seiner Dissertation neue Methoden zur Messung von Entscheidungszeitpunkten der Parteiwahl vor und schließt damit eine wesentliche Forschungslücke. Während bis in die 1980er Jahre hinein weite Teile der wahlberechtigten Bevölkerung als Stammwähler der beiden so genannten großen Volksparteien CDU und SPD galten, wird seitdem eine stetig abnehmende Parteiidentifikation (Dealignment) diagnostiziert, die mit einer erhöhten Wechselbereitschaft der Wähler zu anderen Parteien korrespondiert. Daraus folgt, dass sich aufgrund einer veränderten, ambivalenteren Entscheidungssituation der Entscheidungszeitpunkt von ehemals früh (etliche Monate vor der Wahl) auf spät (wenige Tage vor der Wahl) verschoben hat. Konventionelle Modelle der Wahlentscheidung zum Beispiel auf Grundlage einer Parteiidentifikation sind heutzutage jedoch nur schlecht in der Lage, die Beweggründe für späte Wahlentscheidungen zu erklären. Galten Spätentscheider bisher als wenig politisch interessiert, so zeigt Plischke nun, dass ihren Entscheidungen durchaus sehr rationale Prozesse zugrunde liegen können. Er führt dies vor allem auf eine heutzutage wesentlich ausdifferenziertere Parteienlandschaft zurück. Insbesondere weil Spätentscheider als ein sehr unberechenbares und zugleich wahlentscheidendes Wählersegment gelten, dürfte Plischkes Arbeit für die Wahlkampfforschung von großem Interesse sein.

Für sogenannte Frühentscheider widerlegt er zudem die bisherige Annahme, dass diese gegenüber jeglicher persuasiver Wahlkampfkommunikation immun seien und weist stattdessen nach, dass sie theoretisch durchaus beeinflussbar sind, jedoch faktisch nicht beeinflusst wurden. Dies ist insofern interessant, als es ein neues Licht auf zukünftige Wahlkampfstrategien wirft, weil Frühentscheider nicht mehr länger als wechselresistent gelten 
dürften. Im Gegenteil: Plischke bezeichnet als ein zentrales Ergebnis seiner Arbeit die Tatsache, dass Wähler in ambivalenten Entscheidungssituationen sehr sensibel auf Informationen reagieren. Hieraus wird deutlich, dass - anders als bisher angenommen - die Wirksamkeit der Wahlkampfkommunikation den Zeitpunkt der Wahlentscheidung beeinflusst und nicht umgekehrt.

Diesen Entscheidungszeitpunkt klassifiziert Plischke als eine Grenze, die durch die Abwesenheit dreier Faktoren gekennzeichnet ist: Nichtwahl-Intention, Unentschlossenheit und instabile Wahlintention (S. 83 f). Dieser zunächst redundant wirkenden Erkenntnis liegt seine sehr differenzierte Unterscheidung in unentschlossene, noch nicht endgültig entschlossene sowie endgültig entschlossene Wähler zugrunde, die hilfreich ist, weil die Begriffe „unentschlossen“ und „noch nicht endgültig entschlossen“ bisher synonym verwendet wurden, was die Messung des Zeitpunkts der Parteiwahlentscheidung verzerrt hat.

Der Band ist in drei theoretische und drei empirische Kapitel gegliedert, die in schnörkelloser Sprache geschrieben sind und gründlich lektoriert wurden. Sie enden jeweils mit einer präzisen Zusammenfassung, die es auch eiligen Lesern gestattet, dem Gang der Untersuchung zu folgen.

Theoretisch unterscheidet Plischke zunächst drei Perspektiven auf den Zeitpunkt der Wahlentscheidung. Dies ist erstens der Ansatz affektiver und kognitiver politischer Involvierung - seine Ausführungen nehmen hier explizit Bezug auf Paul F. Lazarsfeld u.a. (1944), zweitens der nach Anthony Downs (1957) entwickelte Informierungsansatz sowie drittens der Ambivalenzansatz, der von so genannten Cross Pressures ausgeht. Schließlich stellt Plischke eine eigens entwickelte Synthese des Involvierungs- und des Ambivalenzansatzes vor, die zeigt, dass sowohl die kognitive und affektive Involvierung als auch das wahrgenommene Ausmaß der Entscheidungssituation als ambivalent den Zeitpunkt der Wahlentscheidung am stärksten beeinflussen (S. $130 \mathrm{ff}$ ).

Für die empirische Prüfung dieser Hypothese stützen sich die Analysen auf die Daten des nicht repräsentativen Wahlkampfpanels der German Longitudinal Election Study (www.gles.eu), die im Rahmen einer siebenwelligen Wiederholungsbefragung zur Bundestagswahl 2009 erhoben wurden. Dabei nimmt Plischke zunächst einen Vergleich von Rückerinnerungs- und Panelmethode vor und prüft, ob beide Methoden valide und zuverlässige Ergebnisse hervorbringen - mit dem Ergebnis, dass für die Identifizierung des Anteils später Wahlentscheidungszeitpunkte die Panelmethode überlegen ist (S. 145).

Als Herzstück der Arbeit bezeichnet der Autor das sechste Kapitel über Bestimmungsfaktoren des Zeitpunkts der Wahlentscheidung. Er stellt hier außerordentliche Methodenkompetenz unter Beweis und gestaltet den Gang seiner Untersuchung unter Zuhilfenahme anschaulicher Graphiken und Tabellen auch für methodisch weniger versierte Leser nachvollziehbar. Die empirischen Analysen bestätigen seine theoretischen Hypothesen insofern, als deutlich wird, dass frühe Wahlentscheidungen zumeist in eindeutigen Entscheidungssituationen von politisch versierten Wählern als Ergebnis rationaler Prozesse getroffen werden (S. 262 f). Politisch Unentschlossene können zu frühen Wahlentscheidungen bewegt werden, wenn ihr Interesse am Wahlkampf früh geweckt werden konnte. Bleibt die verarbeitete Menge an politischen Informationen während des Wahlkampfes gering, fällen politisch weniger versierte Wähler ihre Entscheidungen spät. Cross Pressures wiederum wirken sich zugunsten später Wahlentscheidungen aus (ebenda).

Plischke unterscheidet weiterhin zwischen statischen und dynamischen Determinanten, die den Entscheidungsprozess beeinflussen. Hier hätte es der Arbeit gut getan, diese häufi- 
ger zwischendurch aufzugreifen, um bei weniger involvierten Lesern mehr Verständnis der ansonsten tadellosen Darstellung der Sachzusammenhänge zu erreichen.

Die methodischen Schwierigkeiten der Erfassung von Nichtwählern gestattet es leider bisher nicht, eine vergleichbare Untersuchung auch für dieses Teilelektorat durchzuführen, obwohl es insbesondere vor dem Hintergrund sinkender Beteiligungsraten höchst interessant wäre, Plischkes Arbeit als Referenzpunkt für die Frage heranzuziehen, wann Bürger die Entscheidung für oder gegen die Beteiligung an Wahlen fällen. Dies gilt auch deshalb, weil er nachweist, dass sich die Zunahme später Wahlentscheidungen nicht auf eine späte Mobilisierung der Wähler mit ursprünglicher Nichtwahl-Intention zurückführen lässt.

Plischkes Untersuchungen dürften über den rein politikwissenschaftlichen Kontext hinaus von großem Interesse sein, denn die Frage der Effektivität von Wahlkämpfen wird spätestens mit dem Einsatz von gezieltem „Agenda Setting“ und „Issue Framing“ auch von Kognitionspsychologen, Handlungstheoretikern und Konsumforschern in den Blick genommen.

Sonja Clasing

\section{Wahlsystem- und Wahlverhaltensforschung: innovative Verbindung}

Wagner, Aiko: Die Mikrofundierung von Duvergers Gesetz. Strategisches Wahlverhalten als Wirkungsweise politischer Institutionen (Studien zur Wahl-und Einstellungsforschung, Bd. 24), Nomos Verlagsgesellschaft, Baden-Baden 2013, 277 Seiten, € 44,-.

Der Titel der Dissertationsschrift von Aiko Wagner macht neugierig. Nichts Geringeres als die „Mikrofundierung von Duvergers Gesetz“ wird versprochen. Ausgangsfrage der Arbeit ist, über welche Muster des Wahlverhaltens der als „Duvergers Gesetz“ bekannte Zusammenhang zwischen der Durchlässigkeit des Wahlsystems und der Fragmentierung des Parteiensystems zustande kommt. Im Sinne des methodologischen Individualismus wird also nach der „Mikrofundierung einer Makrotheorie“ gesucht (S. 17). Nachdem Gary W. Cox bereits vor einiger Zeit die Effekte von Wahlsystemen auf Kandidaten umfassend untersucht hat ${ }^{1}$, richtet diese Arbeit den Blick auf die strategischen Anreize für Wähler. Folgerichtig wird eine Verknüpfung der Wahlsystem- und der Wahlverhaltensforschung angestrebt.

Der Autor beginnt mit einer sehr guten Wiedergabe des Forschungsstandes, die jedem nur wärmstens empfohlen werden kann, der sich insbesondere in die Wahlsystemforschung einlesen möchte (Kapitel 2). Bei den Theorien des Wahlverhaltens wird der Schwerpunkt auf den rational-choice-Ansatz gelegt, da der psychologische Effekt von Wahlsystemen ebenfalls auf Kosten-Nutzen-Abwägungen der Akteure beruht. Im dritten Kapitel geht Wagner über die bestehende Forschung hinaus, verknüpft beide Stränge überzeugend und generiert Hypothesen. Als Erklärungsfaktoren des Wahlverhaltens dienen zunächst die Parteiidentifikation, die ideologische Nähe und die Kandidateneinschätzung. Die zentrale Hy-

1 Vgl. Gary W. Cox, Making Votes Count. Strategic Coordination in the World's Electoral Systems, Cambridge 1997. 